Post ural miscl e act i vity patterns during standing at rest and on an oscillating fl oor

\begin{tabular}{|l|l|}
\hline 著者 & $\begin{array}{l}\text { Fuj i war a Kat suo, Toyana Hi roshi , K yot a Takeo, } \\
\text { Needa Kaor u }\end{array}$ \\
\hline $\begin{array}{l}\text { j our nal or } \\
\text { publ i cat i on t i t l e }\end{array}$ & Jour nal of El ect r ony ogr aphy and Ki nesi ol ogy \\
\hline vol une & 16 \\
\hline number & 5 \\
\hline page range & $448-457$ \\
\hline year & $2006-10-01$ \\
\hline URL & ht t p: //hdl . handl e. net /2297/2953 \\
\hline
\end{tabular}




\section{Postural muscle activity patterns during standing at rest and on an oscillating floor}

Katsuo Fujiwara *, Hiroshi Toyama, Takeo Kiyota, Kaoru Maeda

Department of Human Movement and Health, Graduate School of Medical Science,

Kanazawa University, 13-1 Takara-machi, Kanazawa 920-8640, Japan

* Corresponding author. Tel.: +81-76-265-2225; fax: +81-76-234-4219

10 E-mail address: fujikatu@med.kanazawa-u.ac.jp (K. Fujiwara)

Keywords: Standing posture; Electromyogram; Anterior/Posterior dominance;

Periodicity; Floor oscillation 


\section{Abstract}

Postural muscle activity pattern was examined in the eyes-closed state after adequate adaptation to floor anteroposterior oscillation. Twenty-three subjects were grouped almost evenly according to dominance of anterior or posterior postural muscles in the trunk and thigh during quiet stance. In the posterior-dominant group, this dominance was maintained at every frequency in most subjects. In the anterior-dominant group, this dominance was maintained in most subjects at 0.1 and 0.5 $\mathrm{Hz}$ but changed to posterior dominance at 1.0 and $1.5 \mathrm{~Hz}$ in about half the subjects.

10 Periodicity of muscle activity was evaluated by EMG amplitude spectrum at the floor oscillation frequency. Periodicity of posterior-dominant muscles in the trunk and thigh increased with increasing oscillatory frequency. In the trunk, the periodicity did not differ significantly between posterior-dominant and anterior-dominant groups. However, in the thigh, periodicity was significantly lower in the anterior-dominant muscles. This

15 was considered to be caused by nonperiodic alternating action of the anterior and posterior muscles. In the lower leg, posterior dominance was observed in quiet stance and at all oscillation frequencies. Periodicity of soleus and gastrocnemius increased at higher frequencies and was higher in gastrocnemius than in soleus. The periodicity 
difference between both muscles decreased with increasing oscillation frequency. 


\section{Introduction}

In quiet stance, the body generally inclines forward with the ankles acting as a pivotal axis, and the posterior muscles of the body are continuously activated. The

5 standing posture is maintained predominantly by posterior muscles, the so-called Guy-and-Derrick principle [2]. Invariably, only the posterior muscles in the lower legs are activated to maintain quiet stance $[1,18,19]$. The relative positional relationship of body segments during this quiet stance is similar under conditions of weightlessness [7], suggesting that relatively stable mechanisms for posture maintenance exist. However, many previous studies have shown that remarkable individual variation exists regarding patterns of postural muscle activity in the trunk and thigh during quiet stance. Continuous activity in the anterior muscles of the trunk has been reported in around $25 \%$ of subjects [2,3]. Continuous activity of the anterior muscles of the thigh is also observed in a few subjects $[1,4,19,20,27,29,32]$. However, it has not been elucidated

15 whether the anterior or posterior postural muscle dominance is a general principle for all postural muscles throughout the body under both static and dynamic conditions.

Postural control is a basic element of voluntary movement and the two processes of upper- and lower-limb movement and postural control are inextricably linked 
$[6,14,21,31]$. When an individual moves the arm while standing at rest, postural muscle activity precedes activity of the arm target muscles [5,28]. We reported that onset timing of postural muscle activation was adjusted depending on quiet standing position $[12,13]$. We therefore presumed that subjective posterior or anterior dominance of postural 5 muscle activity during quiet stance would largely be maintained in dynamic movement.

The present study focused on dominance of postural muscle activity in dynamic postural control during various frequencies of floor oscillation in the anteroposterior direction. We previously reported that the improvement in steadiness of standing posture reached a plateau for a total of 5 minutes during floor oscillation [11]. Moreover,

10 the anteroposterior position of the center of foot pressure (CFP) in the standing posture during floor oscillation shifts forward as oscillation frequency rises [8,22] and the momentum of the anteroposterior direction around the ankle joint changes according to the distance of CFP position from the heel [24]. As CFP position shifts forward, the activity of triceps surae increases while that of tibialis anterior changes very little [10].

15 Therefore, dominance of posterior muscle activity is likely to be more noticeable in the lower leg during floor oscillation. However, this postulated change in trunk and thigh muscle activity has not been discussed in relation to dominance of anterior or posterior muscle activity. 
It has been reported that in the lower leg, periodic muscle activity corresponding to floor oscillation curve increases at relatively higher frequencies of oscillation, with continuous muscle activity not related to the oscillation decreasing in the way between directional changing points of oscillation and periodic ballistic activity increasing where 5 oscillation direction changes [8]. However, the periodicity of the trunk and thigh muscles has not been sufficiently investigated. The trunk muscles may play a functional role in keeping the trunk vertical and maintaining a perceptional axis of the direction of gravity. The thigh muscles, in addition to assisting in this trunk function, adjust the positional relationship between the trunk and leg to maintain the equilibrium of the entire body $[12,17,23]$. Therefore, it is highly possible that the trunk and thigh muscles show different characteristics of activation.

Muscles of the lower leg vary in terms of contraction properties. Soleus (Sol) exhibits continuous activity in quiet stance while gastrocnemius medialis (GcM) demonstrates burst activity, indicating that the former is predominantly supportive and

15 that the latter has a stabilizing element [16,25]. Moreover, inhibition of Sol and facilitation of GcM follows rapid dorsiflexion of the ankle joint in the preparation phase before a phasic arm movement [33], while GcM is activated selectively in running [9]. It has not been elucidated whether the activity and periodicity of these lower leg 
muscles change with gradual increase of the dynamic element of postural control, such as that caused by changing the frequency of floor oscillation.

In this study, we investigated postural muscle activity after adaptation of postural control to floor oscillation, in subjects who exhibited dominant activation of either the 5 anterior (anterior-dominant group) or posterior postural muscles (posterior-dominant group) of the trunk and thigh during quiet stance. Working hypotheses were as follows.

(1) Intergroup differences would be observed in the proportion of subjects exhibiting change in dominance as frequency of floor oscillation was altered.

(2) Regarding dynamic postural control, during floor oscillation at higher 10 frequencies, higher periodicity would occur on the dominant side.

(3) Differences in the muscle activity and periodicity of Sol and GcM would decrease as floor oscillation frequency increased.

\section{Methods}

\subsection{Subjects}

In a preliminarily experiment, electromyograms (EMG) of the anterior and posterior 
muscles of the trunk, thigh, and lower leg were recorded during quiet stance for 61 adults aged 18-25 years. Twenty-three subjects (10 men and 13 women) were selected based on EMG of the postural muscles of the trunk and thigh and grouped according to whether activity of the anterior or posterior muscles was dominant (anterior-dominant 5 group, $\mathrm{n}=12$; posterior-dominant group, $\mathrm{n}=11$ ). For the lower leg, all subjects demonstrated posterior dominance. All subjects were free of any neurologic or orthopedic impairment. Mean values for age, height, weight, and foot length were 20.1 years $(\mathrm{SD}=1.9), 163.0 \mathrm{~cm}(\mathrm{SD}=8.7), 55.0 \mathrm{~kg}(\mathrm{SD}=7.4)$, and $23.9 \mathrm{~cm}(\mathrm{SD}=1.4)$, respectively. Informed consent was obtained from all subjects following explanation of

10 the experimental protocol.

\subsection{Apparatus}

All measurements were taken with subjects standing on a force platform (WAMI,

15 WA1001) mounted on an oscillation table $(60 \mathrm{~cm}$ long, $60 \mathrm{~cm}$ wide, and $27 \mathrm{~cm}$ high, Electric Control Group, PW0198). The platform contained three load cells which recorded CFP position in the anteroposterior direction. The table was sinusoidally oscillated in the anteroposterior direction with $2.5 \mathrm{~cm}$ amplitude and $0.1,0.5,1.0$, and 
1.5 Hz frequencies. An electrical signal from a potentiometer attached to the table was used to detect table position and to measure oscillation frequency using a frequency counter (Advantest Co. Ltd., TR-5822).

Surface electrodes (Medicotest, M-00-S) were arranged in a bipolar configuration to 5 record surface EMG activity of following muscles: rectus abdominis (RA) at the level of the navel, lumbar paraspinal (LP) at the level of the iliac crest, rectus femoris (RF), the long head of biceps femoris (BF), tibialis anterior (TA), GcM, and Sol, all on the right side. After shaving and cleaning the skin with alcohol, electrodes were aligned along the major axis of the muscle with an inter-electrode distance of about $3 \mathrm{~cm}$. Input

10 impedance was reduced to below $5 \mathrm{k} \Omega$. Signals from the electrodes were amplified $(\times 1000-\times 4000)$ and band-pass filtered $(1.6 \mathrm{~Hz}$ to $1.5 \mathrm{kHz})$ with an EMG amplifier (NEC-Sanei, BIOTOP-6R12).

For subsequent analysis, the signals from the potentiometer, force platform, and electrodes were sent to an on-line computer (Iiyama, M533MS) via an A/D converter 15 (Canopus, ADJ-98) at $1000 \mathrm{~Hz}$ with 12-bit resolution. The signal from the force platform was also sent to another on-line computer (NEC, PC9801CV21) via an A/D converter (I/O data, PIO9045) at $20 \mathrm{~Hz}$ with 12-bit resolution, and mean CFP position in the anteroposterior direction was computed. 


\section{3. Procedure}

Subjects performed the following tasks while standing barefoot on the force

5 platform with eyes closed and with the feet $10 \mathrm{~cm}$ apart and parallel. They were instructed to keep their arms hanging freely by their sides and to maintain a relaxed standing posture without intentional flexion of knee and hip joints.

First, this quiet stance was maintained for $10 \mathrm{sec}$, after which the table was oscillated for $60 \mathrm{sec}$, constituting one trial. A set of 5 oscillation trials was performed at

10 each oscillation frequency, which was increased sequentially as described above. Subjects had seated rest periods of one minute between trials and of 3 minutes between sets.

In order to normalize the EMG between each oscillation trial, the following procedure was followed: pivoting at the ankles with the rest of the body kept aligned,

15 subjects gradually leaned forward from the quiet stance over around $5 \mathrm{sec}$, maintained this extreme forward-leaning posture for $3 \mathrm{sec}$, and gradually leaned then backward over around $10 \mathrm{sec}$, before maintaining this extreme backward-leaning posture for a further $3 \mathrm{sec}$. 


\section{4. Data analysis}

Data from the last $5 \mathrm{sec}$ of maintaining quiet stance and the last $50 \mathrm{sec}$ of table oscillation were analyzed.

The mean position of CFP in the anteroposterior direction was calculated as relative distance $(\%)$ from the heel to the total foot length. The mean speed of CFP fluctuation in the anteroposterior direction during floor oscillation was calculated using the weighted 5-point moving average method.

EMGs were band-pass filtered at $20-100 \mathrm{~Hz}$ using the Butterworth method and were then full-wave rectified. Relative muscle activity (RMA) was calculated using the following formula to determine anterior or posterior dominance of muscle activity in the trunk, thigh, and lower leg.

$$
\operatorname{RMA}(\%)=\left(\mathrm{EMG}_{\mathrm{x}}-\mathrm{EMG}_{\min }\right) /\left(\mathrm{EMG}_{\max }-\mathrm{EMG}_{\min }\right) \times 100
$$

15 In this formula, $\mathrm{EMG}_{\mathrm{x}}$ represents the mean $\mathrm{EMG}$ amplitude in each trial; $\mathrm{EMG}_{\mathrm{min}}$, the minimum mean EMG amplitude over a 100-ms period, obtained during quiet stance or forward or backward leaning; and $\mathrm{EMG}_{\max }$, the maximum mean $\mathrm{EMG}$ amplitude, obtained over a 1-sec period while maintaining extreme forward- or backward-leaning 
posture. Muscle activity dominance was determined by comparing relative muscle activities between the anterior and posterior sides in each body segment, and was defined as the side on which relative muscle activity exceeded $110 \%$ of that in the opposite side.

5 Muscle activity exhibited periodicity during maintenance of stance on the oscillation table. In order to compare this periodicity between muscles, each $20 \mathrm{~ms}$ epoch of the rectified EMG trace was averaged and this averaged value was normalized by the EMG mean amplitude across $50 \mathrm{sec}$. Using this normalized data, FFT analysis with frequency resolution at $0.024 \mathrm{~Hz}$ and Hanning windows was performed, and amplitude spectrum 10 at the frequency corresponding to the floor oscillation was calculated (Fig. 1).

For each oscillation cycle, EMG activity pattern in the anterior and posterior muscles was determined for each body segment. Muscle activity lasting at least $67 \mathrm{~ms}$ $(10 \%$ of one cycle time at the highest oscillation frequency $(1.5 \mathrm{~Hz}))$ was identified as an increase in muscle activity.

\section{5. Statistical analysis}


The effects of trial number on mean speed of CFP fluctuation and oscillation frequency in each parameter of EMG activity were assessed by ANOVA or Friedman test depending on whether the significant difference in variance was observed. Post-hoc multiple-comparison analysis was performed using the Newman-Keuls procedures and

5 Tukey test to further examine differences suggested by ANOVA and Friedman test, respectively. The Chi-squared test was used to assess difference in number of subjects whose dominant side changed. Alpha level was set at $p<0.05$. All statistical analyses performed using Excel 2000 (Microsoft Corp.) with Stat Mate III (ATMS Co. Ltd.).

\section{Results}

Representative data for two subjects during the 5th trial at 0.5 and $1.5 \mathrm{~Hz}$ are shown in Fig. 2. The top panel shows an example of posterior dominance of trunk and thigh muscle activity during quiet stance and the bottom panel shows an example of anterior

15 dominance. In both subjects, posterior dominance was observed for the lower leg, and the mean position of CFP during floor oscillation tended to shift forward compared with that during quiet stance. However, individual differences were observed in the muscle activities of the trunk and thigh during floor oscillation. 
In the posterior-dominant subject, this dominance was maintained in the trunk and thigh during floor oscillation, and activity of these muscles tended to increase at higher oscillation frequencies. At both oscillation frequencies, alternating action of anterior and posterior muscles was rarely observed, and periodicity of activity corresponding to 5 oscillation frequency was observed in the posterior muscles.

In the anterior-dominant subject, dominance of anterior muscle activity was observed in the trunk and thigh at $0.5 \mathrm{~Hz}$. At $1.5 \mathrm{~Hz}$, however, dominance in the thigh changed from anterior to posterior, and alternating action in the thigh muscles was clearly observed.

\subsection{Mean speed and position of CFP fluctuation}

During floor oscillation, mean speed of CFP fluctuation in the anteroposterior direction decreased rapidly until the 3 rd trial for each frequency $\left(F_{4,88}>5.54, p<0.001\right)$,

15 exhibiting no significant change thereafter (Fig. 3). The following descriptions therefore all refer to data collected during the 5th trial.

Fig. 4 shows the mean position and fluctuation range $( \pm \mathrm{SD})$ of $\mathrm{CFP}$ in the anteroposterior direction during quiet stance and floor oscillation in the 
posterior-dominant and anterior-dominant groups. No significant intergroup difference in mean position was found for either the trunk or thigh. At $0.5 \mathrm{~Hz}$ and above, mean position of CFP shifted anteriorly compared with that when standing at rest (posterior-dominant group: $F_{4,40}=3.89, p<0.01$; anterior-dominant group: $F_{4,44}=20.60$, $5 \quad p<0.001)$. Moreover, range of fluctuation increased only in the forward direction.

\section{2. Anterior/posterior dominance of muscle activity during floor oscillation}

Table 1 shows the number of subjects for whom dominant side was maintained

10 during floor oscillation. Dominant side remained unchanged in all of the trunk posterior-dominant group. In the trunk anterior-dominant group, dominant side was maintained in 10 subjects at $0.5 \mathrm{~Hz}$ and in 8 subjects at 1.0 and $1.5 \mathrm{~Hz}$. In the thigh posterior-dominant group, dominant side was maintained in 9 subjects at $0.5 \mathrm{~Hz}$ and in 10 subjects at 1.0 and $1.5 \mathrm{~Hz}$. Significantly fewer subjects in the thigh anterior-

15 dominant group maintained this dominance at $1.0 \mathrm{~Hz}$ when compared to the thigh posterior-dominant group ( 6 vs. $10, \chi_{1}^{2}=4.54, p<0.05$ ). In the lower leg, posterior dominance was maintained by all subjects throughout oscillation.

Each above-mentioned group was further analyzed according to whether or not 
dominance was maintained through all oscillation frequencies. Fig. 5 shows relative muscle activity among subjects who maintained posterior dominance. All subjects in the trunk posterior-dominant group maintained this pattern, and posterior (LP) activity was significantly greater at $0.5 \mathrm{~Hz}$ and above than during standing at rest $\left(F_{4,40}=20.93, p<\right.$ 5 0.001). Posterior dominance of the thigh muscles was maintained in 9 of 11 subjects. In these 9 subjects, activities of posterior and anterior muscles (BF and RF) were significantly greater at 1.0 and $1.5 \mathrm{~Hz}$ than during standing at rest $\left(F_{4,32}>4.35, p<\right.$ 0.01). In the lower leg, the activities of GcM and Sol were significantly greater at 0.5 $\mathrm{Hz}$ and above than during standing at rest $\left(F_{4,88}>13.28, p<0.001\right)$. Activity of GcM

10 was significantly greater than that of Sol only at $0.5 \mathrm{~Hz}(p<0.05)$. Dominance changed in only 2 subjects with posterior dominance of the thigh, and these subjects demonstrated slightly higher activities of the anterior muscles than of the posterior muscles at higher oscillation frequencies.

Fig. 6 shows relative muscle activity in subjects who maintained anterior dominance 15 (left panel) and in those in whom anterior dominance changed to posterior dominance (right panel). In the trunk and thigh anterior-dominant group, dominant side was maintained in 7 and 5 subjects, respectively, and no significant change in activities of anterior and posterior muscles was seen across all oscillation frequencies. 
In subjects in whom anterior dominance of the trunk and thigh changed to posterior dominance ( $\mathrm{n}=5$ and 7 , respectively), activity of the posterior muscles significantly increased in the higher oscillation frequencies (trunk anterior-dominant group: $F_{4,16}=$ 16.21, $p<0.001$; thigh anterior-dominant group: $\left.F_{4,4}=7.13, p<0.05\right)$, while activity of

5 the anterior muscles showed no significant change. In the trunk anterior-dominant group, activity of the posterior muscles was significantly greater than that of anterior muscles at $1.5 \mathrm{~Hz}(p<0.05)$.

\section{3. Periodicity of muscle activity}

Fig. 7 shows the amplitude spectrum of dominant muscle activation during floor oscillation. In the trunk and thigh, the periodicity of posterior muscle activity increased significantly with increasing oscillation frequency (trunk: $F_{3,50}=8.22, p<0.001$, thigh: $\left.F_{3,50}=3.80, p<0.05\right)$, while that of anterior muscles, particularly in the thigh, was higher at $0.5 \mathrm{~Hz}$ and above than at $0.1 \mathrm{~Hz}$ (thigh: $F_{3,34}=5.00, p<0.01$ ), and showed no significant difference when frequency exceeded $0.5 \mathrm{~Hz}$. The periodicity of posterior muscles at 1.0 and $1.5 \mathrm{~Hz}$ was significantly higher than that of anterior muscles $(p<$ $0.01)$ in the thigh. The periodicity of posterior dominant muscles in the trunk was lower 
than that of thigh $\left(F_{1,100}=11.43, p<0.001\right)$; this difference was significant at $1.5 \mathrm{~Hz}$.

In the lower leg, the periodicity of GcM and Sol was significantly higher at $0.5 \mathrm{~Hz}$ and above than at $0.1 \mathrm{~Hz}\left(F_{3,66}>9.32, p<0.001\right)$. The periodicity of GcM was significantly higher than that of Sol at all frequencies $(p<0.01)$ and that of GcM was maximal at $0.5 \mathrm{~Hz}$. The periodicity of Sol was significantly higher at $1.5 \mathrm{~Hz}$ than at 0.5 $\mathrm{Hz}(p<0.05)$. The difference in periodicity between both muscles was greatest at 0.5 $\mathrm{Hz}$ and decreased significantly at $1.5 \mathrm{~Hz}\left(F_{3,66}>7.75, p<0.001\right)$.

The pattern of muscle activity in each oscillation cycle was categorized into anterior or posterior dominant, alternating, and simultaneous. The number of cycles analyzed for each subject was $5,25,50$, and 75 cycles at $0.1,0.5,1.0$, and $1.5 \mathrm{~Hz}$, respectively. Table 2 shows the relative number (\%) of cycles in which alternating activity was observed. In the trunk and thigh posterior-dominant group, only posterior activity was observed in the great majority of cycles (particularly in the trunk), alternating activity was observed in few cycles, and simultaneous activity was observed very infrequently $(0-2 \%$ of 15 cycles). In the trunk and thigh anterior-dominant group, alternating activity was observed in comparatively more oscillation cycles (29-43\%) at $0.5 \mathrm{~Hz}$ and above. In the lower leg, for which all subjects demonstrated posterior dominance, alternating activity was observed at similar levels to the trunk and thigh anterior-dominant group, however, 
most of the anterior muscle (TA) activity was in the form of bursts (Fig. 2).

\section{Discussion}

Steadiness of standing posture during floor oscillation improved rapidly until the 3rd trial and reached a plateau thereafter, in accordance with a previous study [11]. We therefore considered that this stage reflected adequate postural adaptation and was suitable for determination of postural muscle activity.

In the lower leg, dominance of the posterior muscles was maintained at every oscillation frequency in all subjects and this dominance became more prominent as frequency of floor oscillation was increased. During floor oscillation, forward shift of

15 CFP was observed; thought to be related to altered activity of the posterior muscles in the lower leg. An increase of acceleration in the anteroposterior direction corresponding to the oscillation frequency causes an increase in the movement amplitude of CFP. If the mean position of CFP does not shift forward, this increase should lead to the activation 
of the anterior leg muscles. The forward shift of the center of mass should inhibit the activity of these anterior muscles. This phenomenon may have a role in preventing falls because the backward base area to maintain backward leaning posture is small due to the location of the ankle joint in the foot.

In the trunk and thigh, dominance of either anterior or posterior muscles was maintained at $0.1 \mathrm{~Hz}$. Therefore it is probable that dominance of muscle activity in the trunk and thigh is maintained in relatively static conditions. At frequencies of $0.5 \mathrm{~Hz}$ and above, dominance was maintained in most subjects in the posterior-dominant group. In addition, dominance of the posterior muscles became more prominent in these 10 subjects as floor oscillation frequency was increased. This probably indicates increased forward leaning of the trunk and thigh caused by forward shift of CFP during floor oscillation. These phenomena suggest that postural control based on dominance of the posterior postural muscles does not preclude the necessity for forward shift of CFP.

In the trunk and thigh anterior-dominant group, anterior muscle dominance was 15 maintained in more than $80 \%$ of subjects at $0.5 \mathrm{~Hz}$, and in over half the subjects at 1.0 and 1.5 Hz. This suggests that maintenance of standing posture using dominance of either posterior or anterior postural muscles is a comparatively basic requirement of postural control, even at relatively high oscillation frequencies. 
However, change in dominance was also observed, albeit in less than half the subjects. This individual difference in dominance would suggest complicated factors related to selection of active muscles during floor oscillation at high frequency. These factors are postulated to include 1) the requirement for forward shift of CFP, 2)

5 maintenance of a perceptional axis of the direction of gravity by keeping the trunk vertical, and 3) adjustment of positional relationship between the trunk and leg to maintain the body equilibrium $[12,17,23]$.

\subsection{Periodicity of activity in the dominant muscles}

Low periodicity of muscle activity in the dominant side was observed in every measured region at $0.1 \mathrm{~Hz}$. This muscle activity is likely to be strongly related to the antigravitational supportive functions involved in maintaining the standing posture, which do not necessarily correspond to the lower oscillation frequencies. oscillation frequency. In addition, the main pattern of muscle activity during one oscillation period in the posterior-dominant group was posterior dominance only, with alternating activity occurring very rarely. Hence, in the posterior-dominant group, 
postural muscle activity intensified at higher frequencies in a contraction-relaxation manner according to the Guy-and-Derrick principle.

Periodicity of posterior-dominant muscles in the trunk, particularly at $1.5 \mathrm{~Hz}$, was lower than that in the thigh. The incidence of alternating activity of the posterior trunk 5 muscles was extremely low at every frequency. This suggests that the postural supportive element of posterior-dominant muscle activity is stronger in the trunk than in the thigh, particularly at $1.5 \mathrm{~Hz}$. In the trunk, periodicity of the anterior muscles was similar to that of the posterior muscles at every frequency. In the thigh, periodicity of the anterior muscles was lower than that of the posterior muscles at 1.0 and $1.5 \mathrm{~Hz}$. In

10 both anterior- and posterior-dominant groups of the thigh, alternating activity was observed relatively more often at 1.0 and $1.5 \mathrm{~Hz}$. This suggests that when anterior muscle activation is dominant, particularly in the thigh, the periodicity of the anterior muscles at 1.0 and $1.5 \mathrm{~Hz}$ falls with increasing nonperiodic alternate action of anterior and posterior muscles.

\subsection{Activation of GcM and Sol}

Relative muscle activity of GcM and Sol increased at oscillation frequencies of 0.5 
$\mathrm{Hz}$ and above, with GcM tending to show higher activation. However, difference in relative muscle activity between these muscles decreased with increasing oscillation frequency. A similar pattern was observed for the periodicity of GcM and Sol activity. These results suggest that, at $0.5 \mathrm{~Hz}$, GcM plays a relatively important role in the 5 control of torque around the foot joint, and that Sol contributes strongly to the maintenance of the joint stiffness by generating relatively constant tension [15]. This is related to the fact that the periodic change in the floor oscillation was predictable, and that activation of GcM is selectively controlled by the supraspinal nervous system $[9,33]$. It is conceivable that the dynamic element of postural control increases in place

10 of the supportive element according to the increase in floor oscillation frequency. The increase in the periodicity and activity of Sol at high frequencies in the present study indicates that, in contrast to GcM, dynamic activity of Sol is relatively enhanced. In the seated position, GcM is reported to have a close relationship with high-speed eccentric contraction, in marked contrast to Sol [26,30]. It can be inferred from these findings that

15 the difference in Sol activity depends on whether maintenance of posture is the main task.

\section{References}


[1] Åkerblom B. Standing and sitting posture. A. B. Nordiska Bookhandeln: Stockholm, 1948

[2] Asmussen E. The weight-carrying function of the human spine. Acta Orthop. Scandinav. 1960; 29: 276-90

[3] Asmussen E, Klausen K. Form and function of the erect human spine. Clin. Orthopaedics 1962; 25: 55-63

[4] Basmajian JV, Bentzon JW. An electromyographic study of certain muscles of the leg and foot in the standing position. Surg. Gynec. Obst. 1954; 98: 662-66

10 [5] Belen'kii VE, Gurfinkel VS, Pal'tsev RI. On elements of control of voluntary movements. Biofizika 1967; 12: 135-41

[6] Brooks VB. The neural basis of motor control. Oxford: Oxford University Press, 1986

[7] Clément G, Gurfinkel VS, Lestienne F, Lipshits MI, Popov KE. Adaptation of postural control to weightlessness. Exp. Brain Res. 1984; 57: 61-72

[8] Dietz V, Trippel M, Ibrahim IK, Berger W. Human stance on a sinusoidally translating platform: balance control by feedforward and feedback mechanisms. Exp. Brain Res. 1993; 93: 352-62 
[9] Duysens J, Tax AAM, van der Doelen B, Trippel M, Dietz V. Selective activation of human soleus and gastrocnemius in reflex responses during walking and running. Exp. Brain Res. 1991; 87: 193-204

[10] Fujiwara K, Ikegami H, Okada M. The relationship between the postural stability and the relative muscle load of lower limbs in upright stance. Bull. Health \& Sports Sci., Univ. of Tsukuba. 1985; 8: 165-71

[11] Fujiwara K, Toyama H, Asai H, Yamashina T. Development of adaptability of postural control during floor vibration. In: Brandt T, Paulus W, Bles W, Dieterich M, editors. Disorder of posture and gait 1990. New York: Georg Thieme Verlag Stuttgart, 1990: 185-89

[12] Fujiwara K, Maeda K, Toyama H. Influences of illusionary position perception on anticipatory postural control associated with arm flexion. Electromyogr. Kinesiol. 2003; 13: 509-17

[13] Fujiwara K, Toyama H, Kunita K. Anticipatory activation of postural muscles associated with bilateral arm flexion in subjects with different quiet standing positions. Gait and Posture 2003; 17; 254-63

[14] Fukuda T. Stato-kinetic reflexes in equilibrium and movement. Tokyo: Igaku-Shoin, 1957 
[15] Gurfinkel VS, Kots YM, Shik ML. Postural regulation in man. Moscow: Science Publishing House, 1965

[16] Gurfinkel VS, Lipshits MI, Mori S, Popov KE. Interaction of the central program for postural control and inherent reflex mechanisms such as the stretch reflex during standing. Aggressologie 1983; 24: 63-64

[17] Gurfinkel VS, Levik YS, Popov KE, Smetanin BN, Shlikov VY. Body scheme in the control of postural activity. In: Gurfinkel VS, Loffe ME, Massion J, Roll JP, editors. Stance and Motion. New York: Plenum Press, 1988: 185-93

[18] Hellebrandt FA, Tepper RH. The location of the cardinal anatomical orientation planes passing through the center of weight in young adult women. Am. J. Physiol. 1973; 121: 465-70

[19] Joseph J, Nightingale A. Electromyography of muscles of posture: Thigh muscles in males. J. Physiol. 1954; 126: 81-85

[20] Joseph J, Williams PL. Electromyography of certain hip muscles. J. Anat. 1957; 91: 286-94

[21] Magnus R. Some results of studies in the physiology of posture. Lancet 1926; 2: $531-88$

[22] Maki BE, Ostrovski G. Scaling of postural responses to transient and continuous 
perturbations. Gait and Posture 1993; 1: 93-104

[23] Massion J. Movement, posture and equilibrium: interaction and coordination. Prog. Neurobiol. 1992; 38: 35-56

[24] Murray MP, Center of gravity, center pressure and supportive forces during human activities. J. Appl. Physiol. 1967; 23: 831-38

[25] Nardone A, Schieppati M. Postural adjustments associated with voluntary contraction of leg muscles in standing man. Exp. Brain Res. 1988; 69: 469-80

[26] Nardone A, Schieppati M. Shift of activity from slow to fast muscle during voluntary lengthening contractions of the triceps surae muscles in humans. J.

$10 \quad$ Physiol. $1988 ; 395: 363-81$

[27] Oota Y. Electromyography in study of clinical kinesiology. Kyushu J. M. Sc. 1956; 7: 75-91

[28] Pal'tsev RI, El'ner AN. Change in the functional state of the segmental apparatus of the spinal cord under the influence of sound stimuli and its role in voluntary movement. Biofizika 1967; 12: 1219-26

[29] Portnoy H, Morin F. Electromyographic study of postural muscles in various positions and movements. Am. J. Physiol. 1956; 186: 122-26

[30] Romanò C, Schieppati M. Reflex excitability of human soleus motoneurones 
during voluntary shortening or lengthening contractions. J. Physiol. 1987; 390: $271-84$

[31] Sherrington CS. The integrative action of nervous system. 2nd ed. New Haven: Yale Univ. Press, 1947

5 [32] Wheatley MD, Jahnke WD. Electromyographic study of the superficial thigh and hip muscles in normal individuals. Arch. Phys. Med. 1951; 32: 508-15

[33] Woollacott MH, Bonnet M, Yabe K. Preparatory process for anticipatory postural adjustments: modulation of leg muscles reflex pathways during preparation for arm movements in standing man. Exp. Brain Res. 1984; 55: 263-71 


\section{Figure legends}

Fig. 1. FFT analysis of the periodicity of muscle activity. (A) Normalized EMG of Sol from 27 to $35 \mathrm{sec}$ in the 5 th trial at $0.5 \mathrm{~Hz}$ is presented. (B) The maximum amplitude spectrum is observed at the frequency corresponding to the floor oscillation.

Fig. 2. Typical examples of CFP position and muscle activity in 2 subjects. Data from 20 to $30 \mathrm{sec}$ during the 5 th trial at 0.5 and $1.5 \mathrm{~Hz}$ are presented.

Fig. 3. Mean speed of CFP fluctuation in the anteroposterior direction during floor oscillation. Plotted values are means and standard deviations across all subjects.

Fig. 4. Mean position and fluctuation range of CFP in the anteroposterior direction. Plotted values are means and standard deviations for trunk posterior- and anteriordominant groups.

Fig. 5. Relative activities of posterior and anterior muscles among subjects that maintained posterior dominance. Plotted values are means and standard deviations for posterior and anterior muscles for each body segment. Asterisks indicate significant 
differences $(p<0.05)$.

Fig. 6. Relative muscle activities of posterior and anterior muscles in the anterior-dominant group. Data from those that maintained this dominance and for those

5 in whom dominance changed are presented in the left panel and right panel, respectively. Plotted values are means and standard deviations for posterior and anterior muscles in each body segment. Asterisks indicate significant differences $(p<0.05)$.

Fig. 7. Amplitude spectrum of the dominant muscle groups during floor oscillation.

10 Plotted values are means and standard deviations for posterior and anterior muscles in each body segment. Asterisks indicate significant differences $(p<0.05)$. 
Table 1

Subjects in whom anterior/posterior dominance of muscle activity was maintained during floor oscillation

\begin{tabular}{|c|c|c|c|c|c|c|}
\hline \multirow{2}{*}{$\begin{array}{c}\text { Body } \\
\text { segment }\end{array}$} & \multirow{2}{*}{$\begin{array}{l}\text { Muscle dominance } \\
\text { in QSP }\end{array}$} & \multirow{2}{*}{$\begin{array}{l}\text { Total number } \\
\text { of subjects }\end{array}$} & \multicolumn{4}{|c|}{ Frequency of floor oscillation } \\
\hline & & & $0.1 \mathrm{~Hz}$ & $0.5 \mathrm{~Hz}$ & $1.0 \mathrm{~Hz}$ & $1.5 \mathrm{~Hz}$ \\
\hline \multirow{2}{*}{ Trunk } & posterior & 11 & 11 & 11 & 11 & 11 \\
\hline & anterior & 12 & 12 & 10 & 8 & 8 \\
\hline \multirow{2}{*}{ Thigh } & posterior & 11 & 11 & 9 & 107 & 10 \\
\hline & anterior & 12 & 11 & 10 & $6^{-1}$ & 7 \\
\hline Lower leg & posterior & 23 & 23 & 23 & 23 & 23 \\
\hline
\end{tabular}


Table 2

Relative number (\%) of cycles in which alternating activity was observed

\begin{tabular}{|c|c|c|c|c|c|c|}
\hline \multirow{3}{*}{$\begin{array}{l}\text { Body } \\
\text { segment }\end{array}$} & \multirow{3}{*}{$\begin{array}{c}\text { Muscle dominance } \\
\text { in QSP }\end{array}$} & \multirow{3}{*}{$\begin{array}{l}\text { Total } \\
\text { cycle }\end{array}$} & \multicolumn{4}{|c|}{ Frequency of floor oscillation } \\
\hline & & & $0.1 \mathrm{~Hz}$ & $0.5 \mathrm{~Hz}$ & $1.0 \mathrm{~Hz}$ & $1.5 \mathrm{~Hz}$ \\
\hline & & & 5 & 25 & 50 & 75 \\
\hline \multirow{2}{*}{ Trunk } & posterior & & 0 & 1 & 2 & 3 \\
\hline & anterior & & 12 & $40 *$ & $40 *$ & 29 \\
\hline \multirow{2}{*}{ Thigh } & posterior & & 4 & 18 & 23 & $29 *$ \\
\hline & anterior & & 10 & $43 *$ & 32 & $38 *$ \\
\hline Lower leg & posterior & & 5 & $36^{*}$ & $40^{*}$ & $29 *$ \\
\hline
\end{tabular}


A

Table
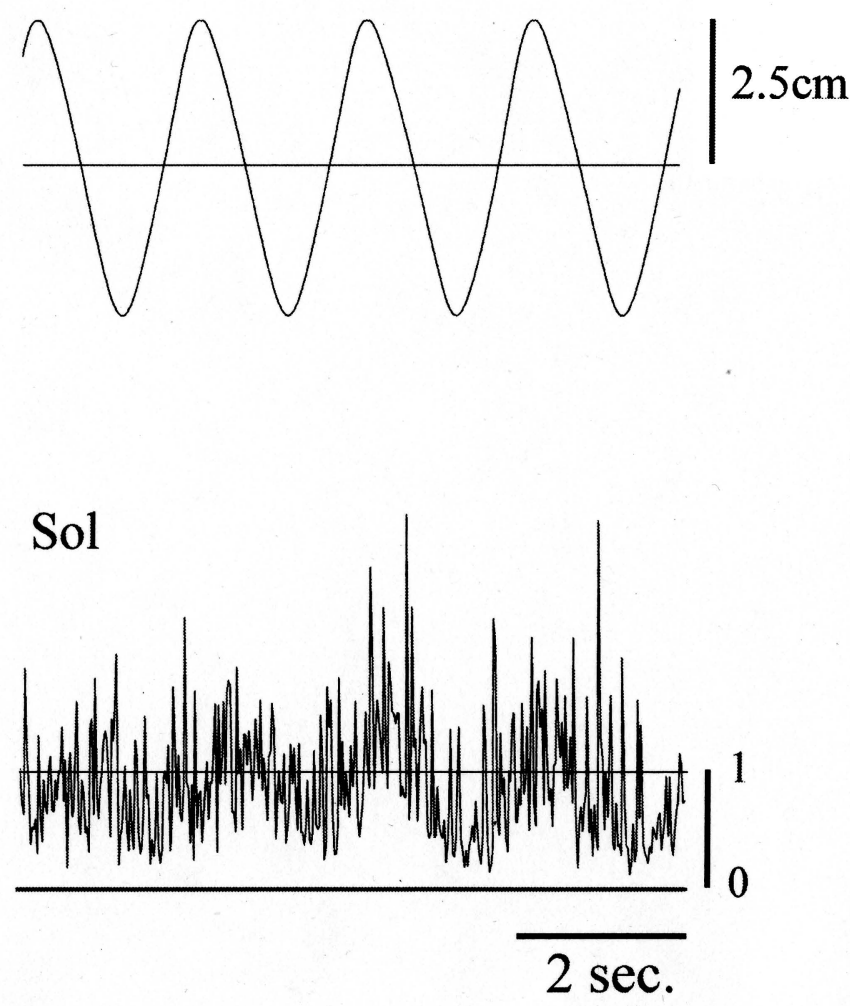

B
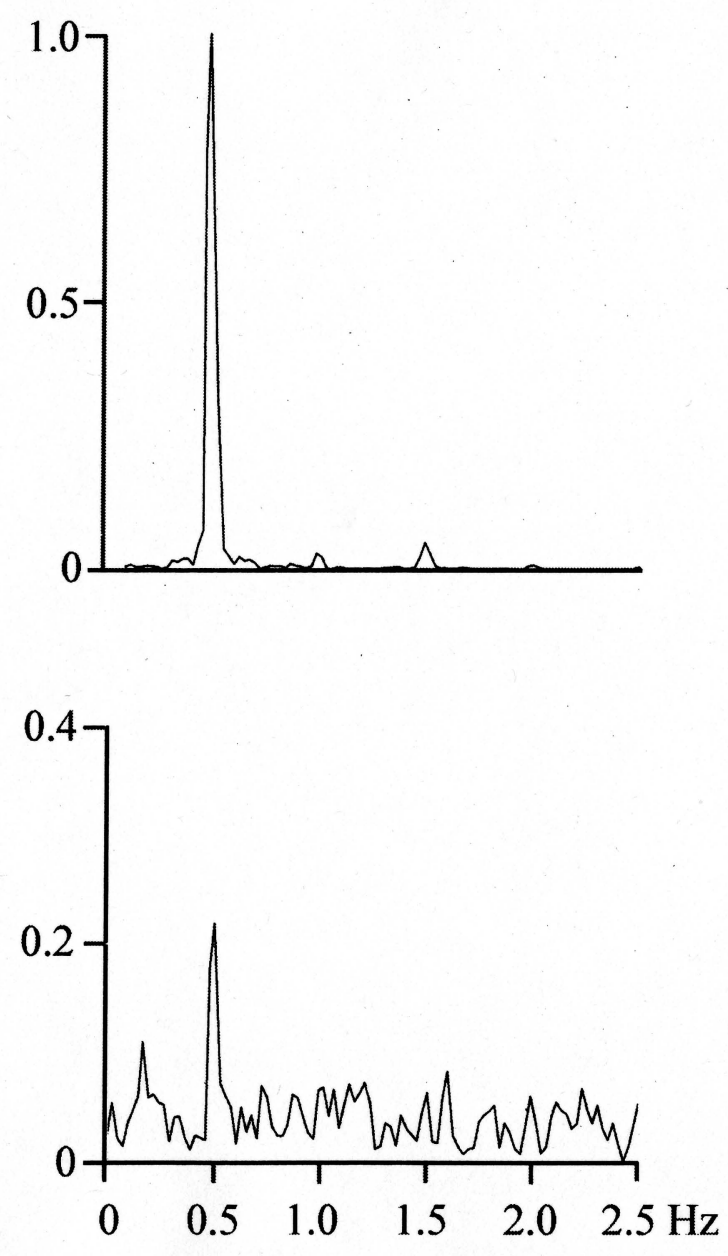
Subj. TK $(0.5 \mathrm{~Hz})$

Table

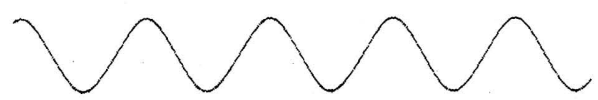

CFP

RA

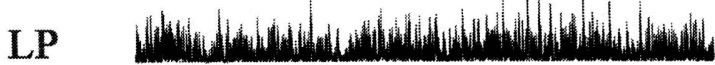

RF

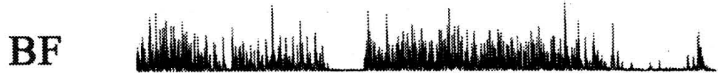

TA

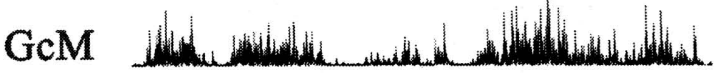

Sol

Subj. KU $\quad(0.5 \mathrm{~Hz})$

Table

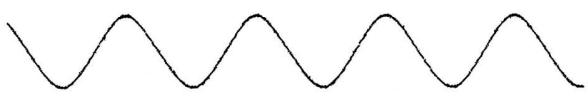

CFP

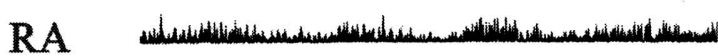

LP

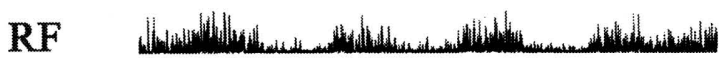

BF

TA

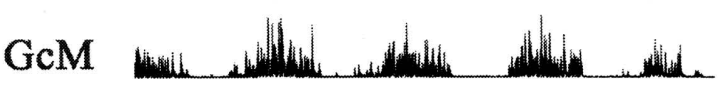

Sol
$(1.5 \mathrm{~Hz})$

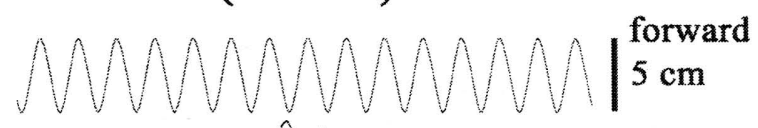

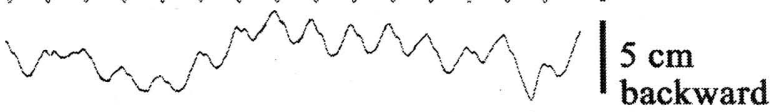
backward $10 \mu \mathrm{V}$

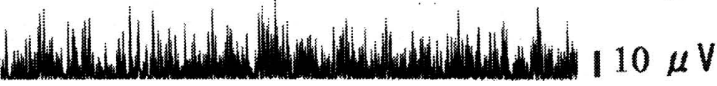

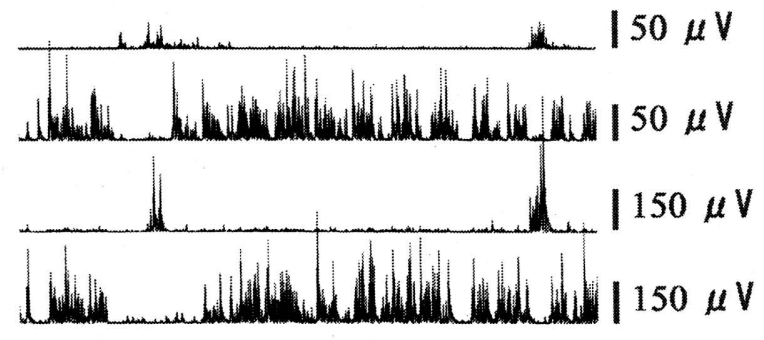

| $150 \mu \mathrm{V}$

\section{$(1.5 \mathrm{~Hz})$}

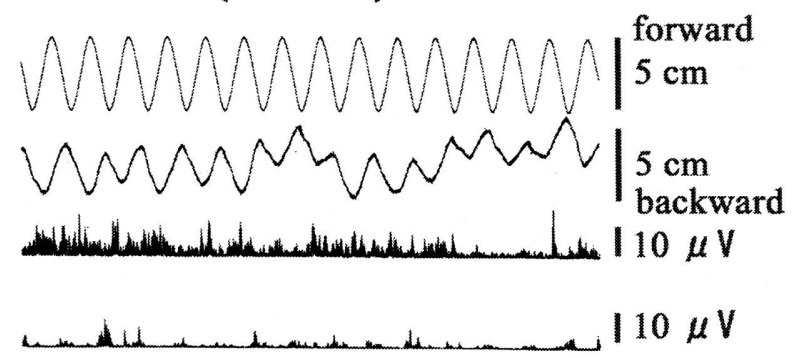

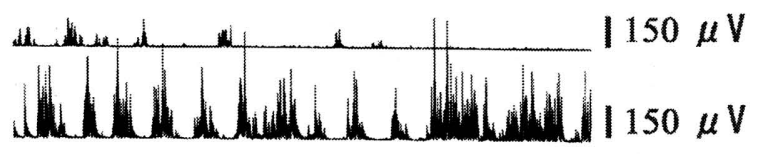

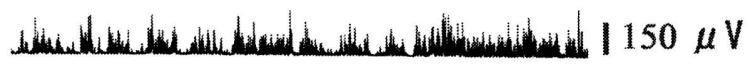




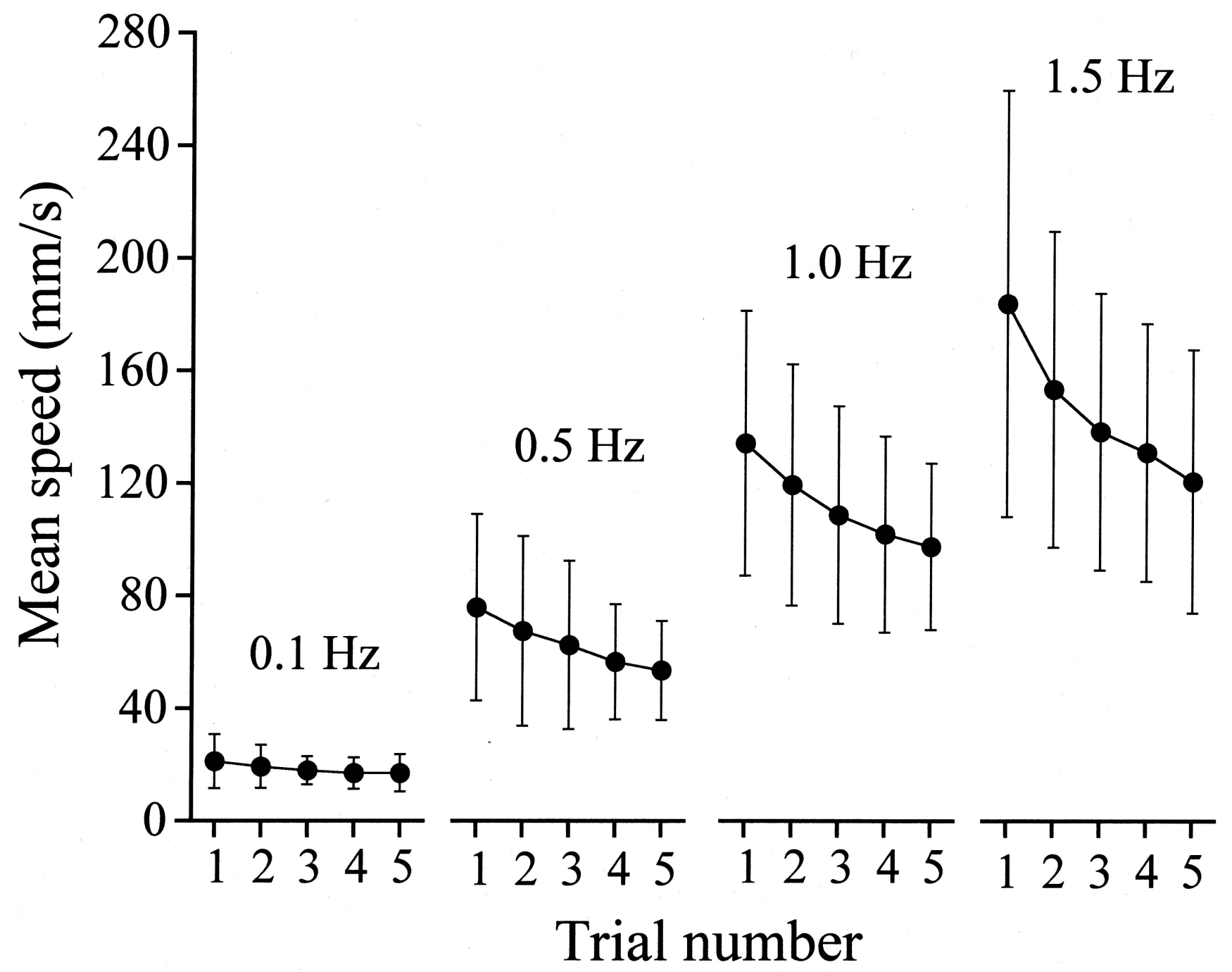




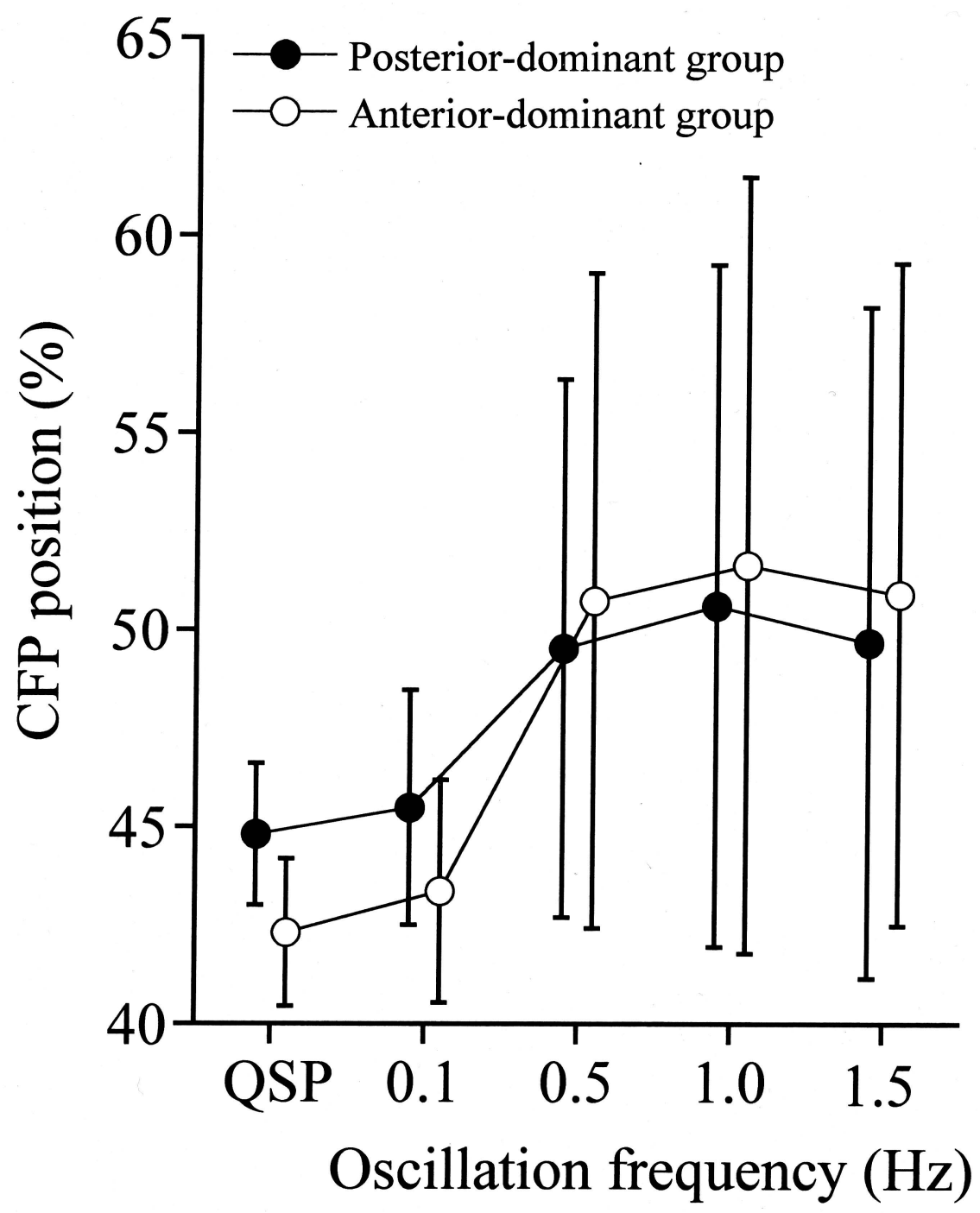




\section{Posterior-dominant group}

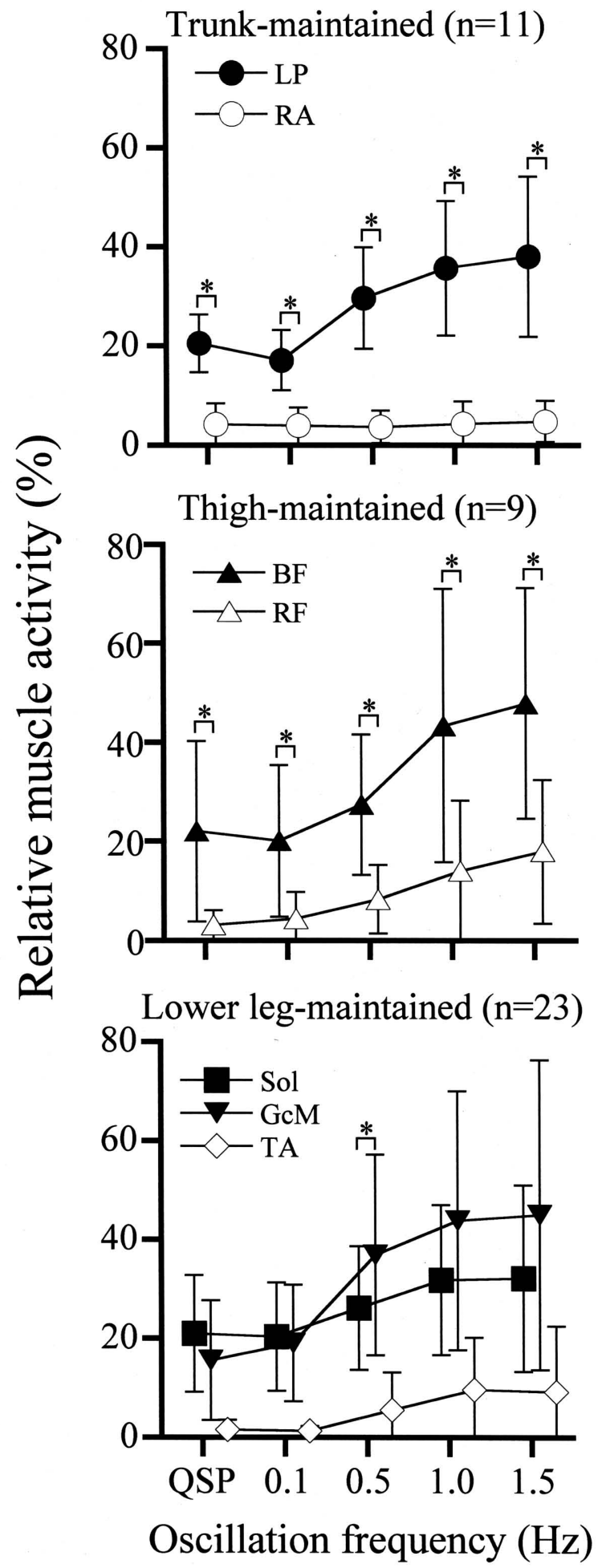




\section{Anterior-dominant group}

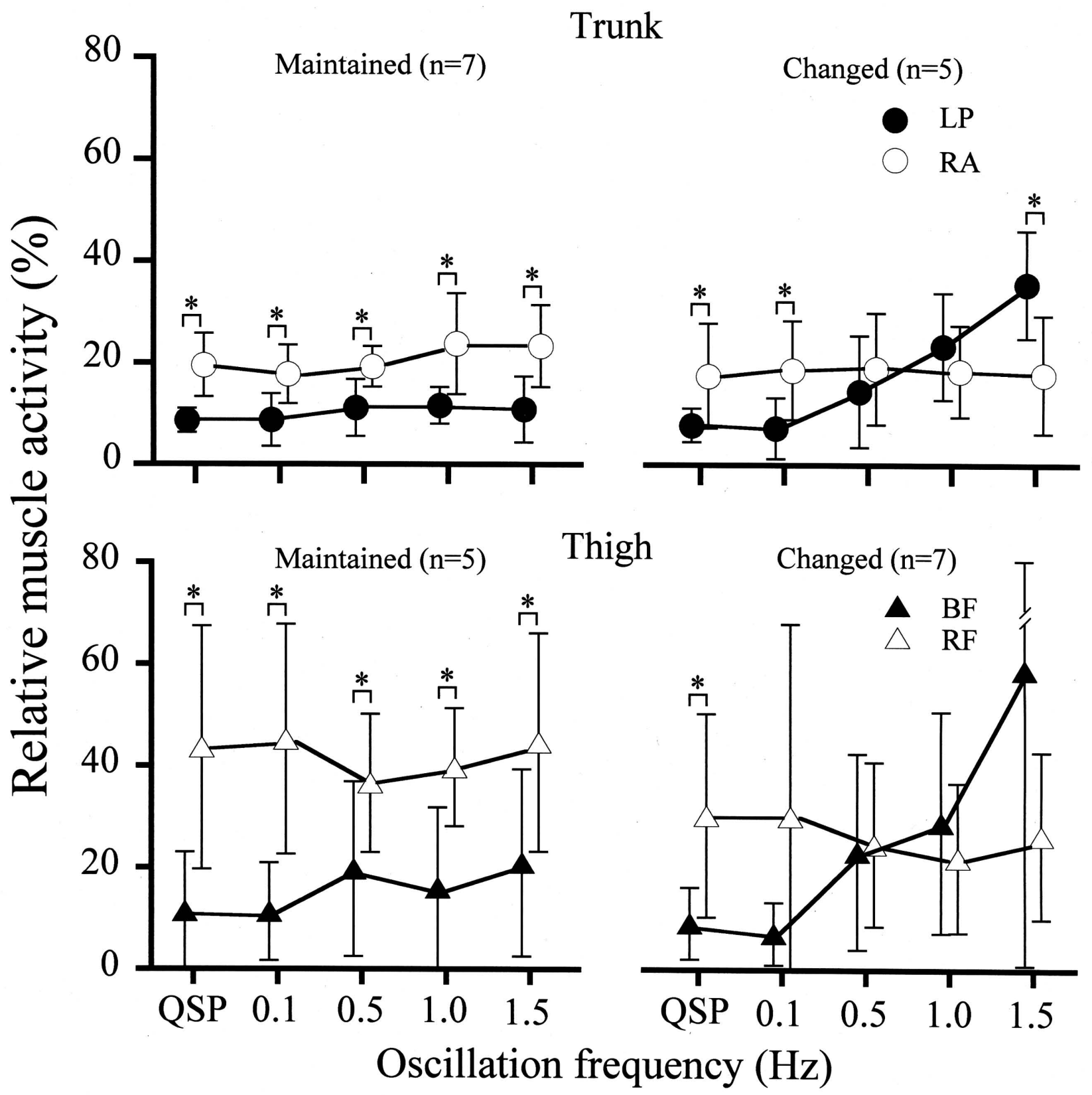



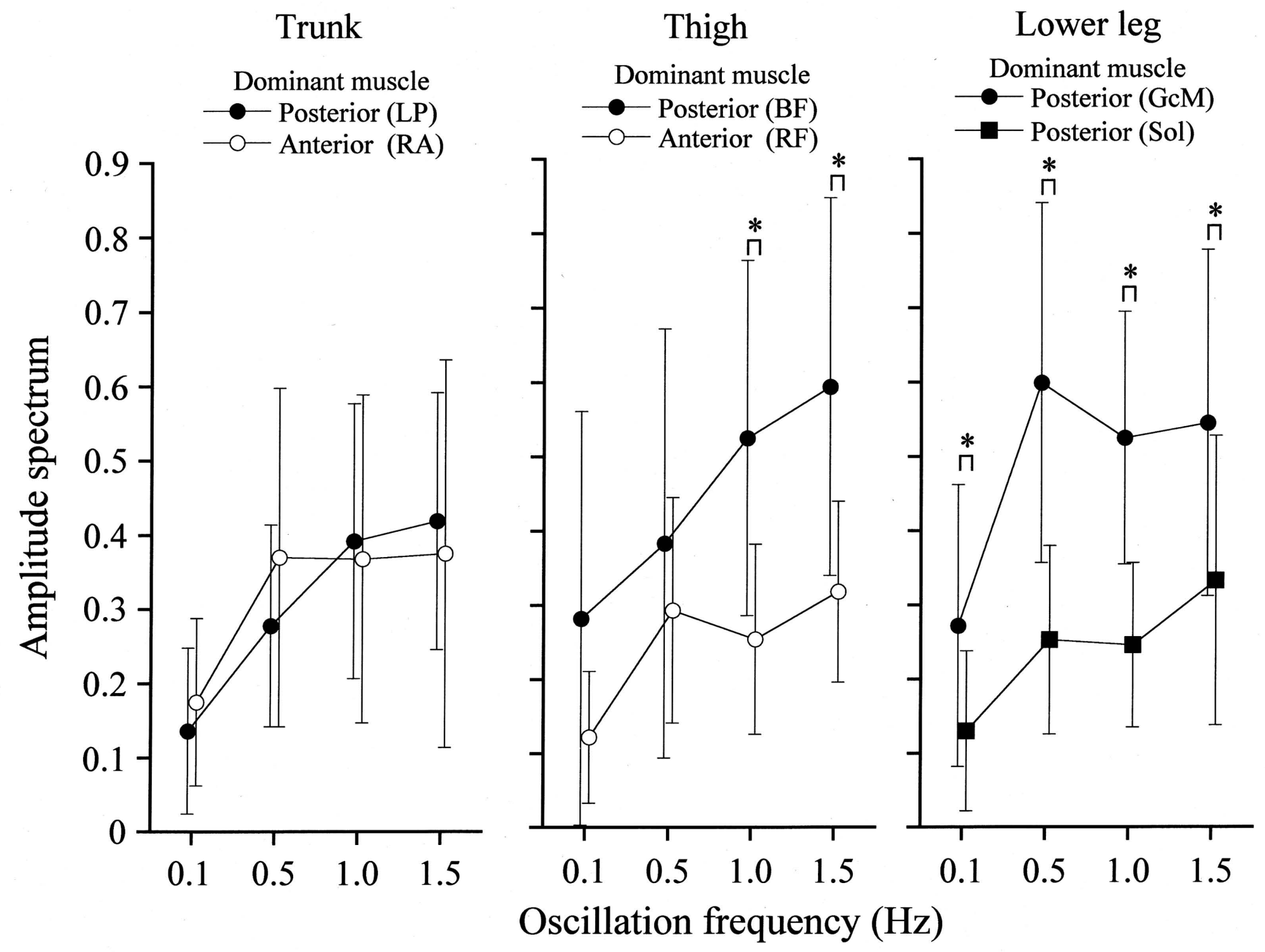